\title{
Overview of Vehicle Networking Platform Based on Cloud
}

\section{Computing}

\author{
Yuan Qinghuii, a, ${ }^{1}$, Feng Guofang ${ }^{2, b}$ and Yuan Qingfei ${ }^{3, c}$ \\ ${ }^{1}$ Binzhou Polytechnic, Binzhou, Shandong Province, 256603 \\ ${ }^{2}$ Sixth Primary Schools in Bincheng, Binzhou, Shandong Province, 256600 \\ ${ }^{3}$ China Offshore Bitumen Co.LTD, Binzhou, Shandong Province China,256600 \\ asdyqh1979@126.com, b48908892@qq.com, cyuanqingfei2007@126.com
}

Keywords: Cloud computing, Vehicle networking, Vehicular terminal

\begin{abstract}
The vehicle networking system platform is a high-tech information product that fully meets the information requirements of automobile manufacturers, logistics enterprises and drivers, which has the features of comprehensive function, simple operation, economy and practicability. It is a monitoring platform for vehicles equipped with intelligent on-board multimedia equipment, which can provide multi-dimensional vehicle operation reports and charts, vehicle location, vehicle security, statistical analysis of the vehicle, message alarm, driving assistance and other functions. Relying on the national Supercomputer Center in Ji'nan's resources, we establish a cloud platform networking system with comprehensive service ability of the car, and carry out a number of cooperation with a number of car manufacturers, and has achieved good market feedback. The vehicle networking system platform can meet the different needs of different customers and improve the modernization level of our vehicle electronic information products.
\end{abstract}

\section{Introduction}

According to the current national automobile industry development rate, by 2020 car ownership is expected to exceed 200 million vehicles, China has become the world's largest car market. The huge car market provides a strong foundation for the growth of China's vehicle networking services, due to the application of vehicle networking and high technology maturity, which makes it become one of the key breakthroughs in the Internet of things. The industrialization prospect and market demand of the vehicle networking system are undoubtedly enormous, ${ }^{[1]}$ With an annual output of 15 million vehicles, with a market share of only $1 \%$, we can realize an annual output of 150 thousand units.

\section{Overview of vehicle networking system platform function}

platform taking vehicle intelligent interconnection and information management as the construction target, based on cloud computing technology, integrated communications, navigation, entertainment, human-computer interaction, vehicle testing as a whole, includes two major components: vehicle information terminal and cloud vehicle networking platform. Vehicle information terminal is a mobile device based on traffic online service system, which provides the following main functions: automobile navigation function, telephone call, short 
message function, video, radio and other entertainment functions, as well as system settings, vehicle information function. Cloud vehicle networking platform is a monitoring platform for vehicles equipped with intelligent on-board multimedia equipment, which can provide multi-dimensional vehicle operation reports and charts, vehicle location, vehicle security, statistical analysis of the vehicle, message alarm, driving assistance and other functions. The concept block diagram of vehicle networking is as Fig.1.

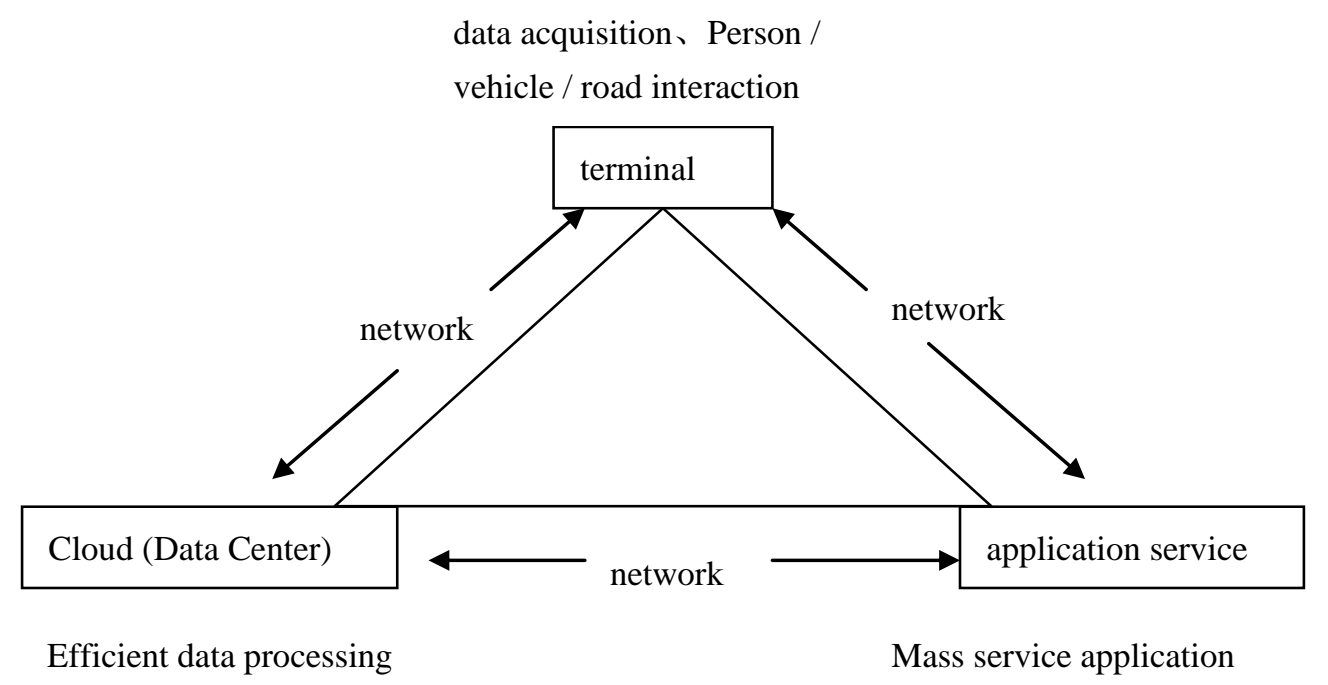

Fig.1 conceptual block diagram of vehicle networking

\section{Vehicle networking system platform composition}

Vehicle networking platform system based on cloud computing and taking vehicle intelligent interconnection and information management as the core, provides the functions of vehicle location, vehicle security, statistics and analysis of the vehicle condition, message alarm and driving assistance. The vehicle location system provides real-time national map positioning and tracking playback services. Vehicle security provides a variety of electronic fence independent settings, in violation of security settings, which will promptly notify the user's mobile phone to ensure vehicle safety anytime and anywhere. Vehicle statistics and analysis can provide multi-dimensional vehicle running statistical reports, and a key condition detection can ensure travel safety before the car. Information alarm provides vehicle abnormalities and promptly notified the driver, the owner, which makes the vehicle longer service life. The problem For a period of time, is summarized in the vehicle anomaly report, which allows the owner to understand the vehicle and respond accordingly. Road aids provide weather information for real-time inspection all over the country.

\section{Online service platform for vehicle networking}

When the user enters the cloud networking online service platform interface, enter the correct user name and password, you can enter the home page of the online service platform. The online service platform interface mainly includes menu area, monitoring and operation 
area, auxiliary display area, real-time display area and other areas. Brief introduction is as follows:

Menu area: you can choose different function modules and related menu commands.

Monitoring and operating area: this area is used primarily to show the location of the vehicle map and other functional areas of operation.

Auxiliary display area: this area is used to display various status data and the corresponding help prompt information.

Real time display area: display the relevant real-time data of vehicle terminal.

\section{The function of the online service system}

It can be divided into eight function modules, which can be entered by menu area:

Monitor home page: after entering the online service platform system, the monitor home page is open by default. The main operations on this page include looking at map positioning view the map, locate, viewing the status of each module, message processing, vehicle location, view real-time data and other information.

Fence Management: including fencing, fencing, fencing, fencing, etc..

Vehicle condition monitoring: display the current vehicle operation information, provide the vehicle's multiple running conditions, and provide the basis for real-time monitoring of vehicles.

Message issued: when the emergency or other situation occurs, it sends the message to the terminal in time, and the terminal will give the feedback to the driver in time.

Condition report: including basic information statistics, fuel consumption statistics, driving statistics, statements, report record query.

Train assist: This module can inquire the weather condition of the vehicle location.

Information management: information management mainly includes information management and parameter setting and includes user basic information and vehicle basic information management. Parameter settings include vehicle location parameter settings, vehicle security parameters, and other parameters set up three parts.

Safe exit: the system will automatically clear user login information and return to the login screen.

\section{Technical innovation point of vehicle networking platform}

Technical innovation point of vehicle networking platform are as follows:

It can collect data in real time and build independent, safe, stable and reliable data acquisition platform and can flexibly configure the bus protocol and acquisition strategy, collect the key data in the process of running vehicles, and provide reliable and reliable reference data for product improvement, upgrading and personalization. VPDN+ unique encryption, authentication and verification technology can ensure the safe and reliable transmission of vehicle information.

Through the storage of the key data information of the vehicle, and the real-time analysis of the vehicle's historical data, we can get the relevant warning information. Through the combination of CAN bus technology and wireless communication technology, stored and analyzed the key data such as failure records, maintenance records and driving records during the process of using vehicles, we set up fatigue driving reminders, road rescue services to 
improve traffic safety guarantee. At the same time, Vehicle security provides a variety of electronic fence independent settings, in violation of security settings, which will promptly notify the user's mobile phone to ensure vehicle safety anytime and anywhere.

Visual vehicle management service, through the combination of GPS satellite positioning technology and wireless mobile Internet technology, provides real-time national map positioning system, vehicle track tracking and playback service.

Big data cloud data analysis can provide multi-dimensional vehicle running statistical reports, and a key condition detection can ensure travel safety before the car. It allows us to keep abreast of the conditions of the car and to minimize the safety risks of the process. Train data analysis helps managers better control logistics operation cost and improve vehicle operation efficiency. Through the use of multi-function reports accumulated vehicle data, we conduct statistical analysis of vehicle driving, fuel consumption, power systems to fully understand the vehicle condition information, which greatly improves the efficiency of the vehicle.

The service system of vehicle fleet management can realize the functions of vehicle management, link management, task dispatch, fuel consumption comparison, route tracking, traffic comparison and so on, and can help the team to manage the home vehicles more fully, provide more applications and reports, and make the vehicle running contrast and analysis more effective.

Intelligent multimedia communication and entertainment system integrates communication, entertainment, Internet access, information, intelligent voice navigation and other functions, and allows owners to easily and conveniently implement voice dialing, voice messaging, voice broadcasting, etc..

Wireless remote control technology can more effectively manage installment vehicles.

Through the two development, API (Application Program Interface)can be seamlessly integrated with the existing production, after-sale management systems.

\section{The technical route of vehicle networking platform}

The vehicle terminal has two directions of communication interface: One connects the on-board CAN bus and collects the data of the vehicle in real time; the other connects the wireless communication network and sends the on-board data to the service center. ${ }^{[3]}$ The wireless data access technology of vehicular terminal adopts GPRS technology, and its communication speed meets the requirements of vehicle monitoring system protocol, and covers a wide area. ${ }^{[2]}$

Vehicle monitoring: accord with the 1939 protocol of the automobile CAN bus, real-time report the vehicle running state data. Users can remotely monitor the vehicle's driving information by computer, including vehicle speed, vehicle mileage, vehicle battery voltage, equipment temperature, communication signal strength, etc. Remote monitoring: the terminal is connected with MIC, and the monitor is realized by calling the terminal number. Remote control: terminal externally is connectted to relay to realize the function of breaking oil and power off.

Vehicle location and track playback: the user can view the running path of the vehicle in the selected period of time by computer playback.

Alarm information: emergency alarm: users press the button to alarm and upload the alarm data to the service center; Power off alarm: after the external power is disconnected, the 
power failure alarm is sent to the service center; Antenna alarm: when the GPS antenna has short circuit or open circuit fault, the terminal will send the alarm to the service center.

Voice navigation: equipped with the latest high German voice navigation system.

$\mathrm{T}+$ service: for the use of vehicles, equipped with different service applications, such as supply information.

Tel: integrated telephone voice system, the use of industry tariffs, no roaming throughout the country.

Voice message: SMS speech reading, to ensure safe driving

Driving record: in line with the Ministry of communications JT/T794-2001, JT/T808-2001 standards.

Entertainment can play 1080p HD movies, MP3/MP4/MP5, radio, e-books, album

Electronic fence: users can limit the car to a certain area, if the vehicle leaves the area, it will alarm.

\section{Conclusions}

Relying on the national Supercomputer Center in Ji'nan's resources, we establish a cloud platform networking system with comprehensive service ability of the car, and carry out a number of cooperation with a number of car manufacturers, and has achieved good market feedback. The vehicle networking system platform can meet the different needs of different customers and improve the modernization level of our vehicle electronic information products. It will become a brand new automotive electronic product, and it will play a positive role in promoting the development of the automotive electronics industry in China. ${ }^{[4]}$

\section{References}

[1] Ma Chunhong. Construction of vehicle networking information service platform based on cloud computing environment [J]. Mapping and spatial geographic information, Vol. 3 (2014), p. 84-87.

[2] Li Hongfeng. Discussion on interactive platform construction plan of electric vehicle vehicle networking service [J]. Power grid and clean energy, Vol. 1 (2016), p. 69-74.

[3] Zhang Jianfeng. Design of remote monitoring system for test vehicle [J]. industrial control computer, Vol. 8 (2009), p. 33-34.

[4] Zhang Yong. Reflections on the development of automotive electronics industry in Wuhu [J]. Journal of Wuhu Vocational Institute of Technology, Vol. 2(2006), p. 88-90. 